\title{
A randomised controlled trial of four weeks versus seven weeks of pulmonary rehabilitation in chronic obstructive pulmonary disease
}

\author{
R H Green, S J Singh, J Williams, M D L Morgan
}

\begin{abstract}
Background-Pulmonary rehabilitation programmes have been shown to improve both exercise tolerance and health status in patients with chronic obstructive pulmonary disease (COPD). The optimal duration for a pulmonary rehabilitation programme is, however, unknown. To assess whether the current pulmonary rehabilitation programme could be shortened a randomised controlled trial was conducted in 44 patients with COPD who were allocated to either a seven week or a four week course.
\end{abstract}

Methods-Patients were randomised to either our standard seven week twice weekly outpatient based programme or a comparable but shortened four week course. They were assessed at baseline and at completion by the Chronic Respiratory Questionnaire (CRQ), the Breathing Problems Questionnaire (BPQ), the incremental shuttle walking test (SWT), and the treadmill endurance test (TET). Results-Twenty one patients (61\% men) of mean (SD) age $68(9.2)$ years and forced expiratory volume in one second $\left(\mathrm{FEV}_{1}\right)$ $1.08(0.4) 1$ completed a conventional seven week course and $23(67 \%$ men) of mean (SD) age $69(8.8)$ years and $F E V_{1}$ $1.03(0.3) 1$ completed a shortened four week course. Patients who completed the seven week rehabilitation programme had greater improvements in all outcome measures than those undertaking the four week course. These differences reached clinical and statistical significance for the total CRQ score, which was the primary outcome variable (mean difference $(95 \%$ confidence intervals (CI) of difference) $-0.61(-0.15$ to -1.08$), p<0.05)$, and the CRQ domains of dyspnoea ( -0.80 (95\% CI -0.13 to -1.48$), p<0.05)$, emotion $(-0.89$ $(95 \%$ CI -0.33 to -1.45$), p<0.005)$, and mastery $(-0.84(95 \%$ CI -0.10 to -1.58$)$, $\mathrm{p}<0.05)$. There were also trends towards greater improvements in exercise assessments in the seven week group but the differences did not reach statistical significance (SWT: mean difference $\mathbf{- 1 6 . 9}$ $(95 \%$ CI 24.8 to -58.6$), p=0.41$; TET: geometric mean difference -1.21 (95\% CI -0.60 to -2.47$), \mathrm{p}=0.56$ ).

Conclusions-A seven week course of pulmonary rehabilitation provides greater benefits to patients than a four week course in terms of improvements in health status. Larger prospective studies are required to determine the optimal duration of a pulmonary rehabilitation programme.

(Thorax 2001;56:143-145)

Keywords: pulmonary rehabilitation; duration; chronic obstructive pulmonary disease

Pulmonary rehabilitation now has an established role in the management of patients with chronic respiratory disease and controlled studies have shown lasting improvements in both health status and exercise capacity. ${ }^{1-5} \mathrm{We}$ have previously reported the benefits to patients completing our seven week programme. ${ }^{6}$ The initial estimates of the optimum length of outpatient rehabilitation programmes were based on the recommendations of the American College of Sports Medicine for training in healthy individuals which suggest that a six week course is required to achieve an aerobic training effect. ${ }^{7}$ For patients with chronic obstructive pulmonary disease (COPD), however, there is little to support seven weeks of training against any other reasonable length of programme. We therefore designed a randomised controlled trial which aimed to determine whether a shortened four week programme would provide equal benefits to patients in terms of improvements in health status and exercise capacity as our traditional seven week course.

\section{Methods}

Forty four patients with COPD (forced expiratory volume in one second $\left(\mathrm{FEV}_{1}\right)<80 \%$, ratio of $\mathrm{FEV}_{1}$ to forced vital capacity (FVC) $<70 \%$, and consistent symptoms) were randomised (using concealed envelopes) to either our conventional seven week rehabilitation programme or a condensed programme of four weeks. At baseline the patients were assessed by the standardised health status measurements-the Chronic Respiratory Questionnaire (CRQ) and the Breathing Problems Questionnaire (BPQ). The CRQ was administered as described by Guyatt et al. ${ }^{8}$ Briefly, four domains are measured: dyspnoea (using self-selected daily activities), fatigue, emotion, and mastery. The domains have 4-7 items each, scored on a scale of 1-7. A change of 0.5 in the mean score per domain (calculated by dividing the overall score by the number of items) has been shown to be associated with a minimally important difference in health status. ${ }^{9}$ The short form $\mathrm{BPQ}$ was administered as previously de- 
scribed. ${ }^{10}$ Patients also completed the incremental shuttle walking test (SWT) using our standard protocol ${ }^{11}$ and the treadmill endurance test. Here subjects walked on a treadmill set at a speed of $60 \%$ of the predicted maximal exercise capacity obtained from the SWT result. ${ }^{12}$ The conventional course of rehabilitation comprised a twice weekly outpatient programme of disease education and exercise training including aerobic walking, general mobility, and strength training exercises for seven weeks. The educational component comprised 14 sessions of discussions and practical demonstrations from a range of health care professionals. Patients randomised to the four week course received an identical but shortened training programme. The four week educational package was modified with patients attending for extended sessions to compensate for the reduction in overall number. On completing the rehabilitation programme the health status and exercise assessments were repeated, the exercise tests by a technician blinded to the study.

The study was approved by the Glenfield Hospital ethics committee and all patients gave written informed consent.

ANALYSIS OF DATA

Baseline results were expressed as mean values with 95\% confidence intervals (CI). The results for the treadmill endurance test were not normally distributed and were therefore expressed as median and interquartile range. To account for the clinically important differences between the two groups observed at baseline, comparisons between the two treatment groups were performed using an analysis of variance model including the baseline values as covariates. For each of the outcome measures the standard residuals had an approximately normal distribution, including those of the treadmill endurance test (TET) following $\log$ transformation. The between treatment group differences were therefore expressed as the geometric mean difference for the TET and the mean difference for all other variables, with $95 \% \mathrm{CI}$ throughout. A $\mathrm{p}$ value of $<0.05$ was considered significant.

\section{Results}

Twenty one patients of mean (SD) age 68 (9.2) years and $\mathrm{FEV}_{1} 1.08(0.4) 1$ were allocated to the seven week course and 23 patients of mean (SD) age 69 (8.8) years and $\mathrm{FEV}_{1} 1.03(0.3) 1$ received the modified four week programme. At baseline there were no significant differences in age, pack years smoked, or $\mathrm{FEV}_{1}$. There were, however, clinically relevant differences in the baseline shuttle walking test distance and in the CRQ domains of fatigue, emotion and mastery (table 1). Following the rehabilitation programme patients who completed the seven week course were found to have greater improvements in each outcome variable than those undertaking the four week course. The differences between the two groups were statistically and clinically significant for the total CRQ score, which was the
Table 1 Mean (SE) baseline demographic data, exercise assessments, and health status

\begin{tabular}{lll}
\hline & $\begin{array}{l}\text { Four week group } \\
(n=23)\end{array}$ & $\begin{array}{l}\text { Seven week group } \\
(n=21)\end{array}$ \\
\hline $\begin{array}{l}\text { No of men } \\
\text { Age }^{\star}\end{array}$ & 14 & 14 \\
Pack years $^{\star}$ & $38(49-84)$ & $68(48-84)$ \\
FEV $(1)$ & $1.03(0.07)$ & $47(15-140)$ \\
SWT (m) & $140.00(13.90)$ & $1.08(0.10)$ \\
TET (min) & $3.44(4.55)$ & $4.58(7.36 .23)$ \\
BPQ & $16.05(0.90)$ & $14.53(0.96)$ \\
CRQ: dyspnoea & $2.65(0.20)$ & $2.63(0.05)$ \\
CRQ: fatigue & $2.86(0.21)$ & $3.42(0.21)$ \\
CRQ: emotion & $3.64(0.22)$ & $4.36(0.20)$ \\
CRQ: mastery & $3.60(0.19)$ & $4.34(0.29)$ \\
CRQ: total & $3.21(0.15)$ & $3.70(0.17)$ \\
\hline
\end{tabular}

$\mathrm{FEV}_{1}=$ forced expiratory volume in one second: SWT $=$ shuttle walking test $; \mathrm{TET}=$ treadmill endurance test $\mathrm{BPQ}=$ Breathing Problems Questionnaire; CRQ $=$ Chronic Respiratory Questionnaire.

${ }^{\star}$ Median (range).

${ }^{\star \star}$ Median (interquartile range).

Table 2 Changes in health status and exercise assessments following rehabilitation: between group comparisons by analysis of variance with baseline measurements included as covariates

\begin{tabular}{lll}
\hline Outcome variable & $\begin{array}{l}\text { Mean difference }(95 \% \text { CI) } \\
\text { between } 7 \text { and } 4 \text { week courses }\end{array}$ & p value \\
\hline BPQ & $1.46(-0.99$ to 3.90$)$ & 0.231 \\
CRQ: total & $-0.61(-1.08$ to -0.15$)$ & $0.011^{\star}$ \\
CRQ: dyspnoea & $-0.80(-1.48$ to -0.13$)$ & $0.021^{\star}$ \\
CRQ: fatigue & $-0.46(-1.24$ to 0.33$)$ & 0.242 \\
CRQ: emotion & $-0.89(-1.45$ to -0.33$)$ & $0.003^{\star}$ \\
CRQ: mastery & $-0.84(-1.58$ to -0.10$)$ & $0.027^{\star}$ \\
SWT (m) & $-16.9(-58.6$ to 24.81$)$ & 0.415 \\
TET (min) & $-1.15(-1.87$ to 1.39$) \dagger$ & 0.56 \\
\hline
\end{tabular}

$\mathrm{BPQ}=$ Breathing Problems Questionnaire; $\mathrm{CRQ}=$ Chronic Respiratory Questionnaire; SWT = shuttle walking test; TET = treadmill endurance test.

${ }^{\star}$ Significant difference.

†Geometric mean difference.

primary outcome variable, and also for the CRQ domains of dyspnoea, emotion, and mastery (table 2).

\section{Discussion}

Since the current evidence shows that pulmonary rehabilitation provides significant benefits to patients, we felt that it would not be ethical to undertake a study that included a placebo arm since a group of patients would thus be denied treatment that we know to be beneficial. Our control group therefore represented our current standard treatment against which the modified programme was compared. Patients undertaking the seven week programme had clinically important improvements in exercise performance and health status consistent with those seen in previous studies. ${ }^{4}$ When compared with the four week course of rehabilitation, the seven week course appears to provide greater benefits to patients with statistically significant and clinically important differences in the primary outcome variable, the total CRQ score, and in three of its individual domains. There was also a trend towards greater improvements in those on the seven week programme for each of the remaining health status and exercise assessments. Failure to show a statistically significant difference in terms of exercise capacity may arise from the relatively small sample size since the study was not powered to demonstrate significant differences in these secondary outcomes. 
There were clinically important baseline differences between the two groups, but the analysis of covariance model allows for these inequalities and we can therefore be confident that we have shown a true difference between the two lengths of treatment. The improvements in those on the four week course were measured at completion of the outpatient programme. It would be interesting to see whether results comparable to those achieved by the seven week programme could be reached by including a further three week period of home exercise training. This would determine whether the observed results represent a "lag effect", with improvements in health status developing after a period of time.

This study was confined to the immediate benefits of pulmonary rehabilitation. A recent randomised controlled trial has reported lasting improvements in exercise capacity, health status, and use of healthcare resources in patients undergoing outpatient pulmonary rehabilitation compared with controls. ${ }^{5}$ It would therefore be interesting to lengthen the follow up period, repeating exercise and health status assessments at six months to see whether the improvements were sustained for a comparable period between the two groups. In terms of the immediately observed benefits, however, the results from this relatively small study lead us to reject our null hypothesis, supporting the theory that the seven week rehabilitation programme is superior. Further larger prospective studies are required to determine the optimal duration for a pulmonary rehabilitation programme.
The authors thank Mr Nick Taub of the Trent Institute for Health Services Research for his statistical advice.

1 Goldstein RS, Gort EH, Stubbing D, et al. Randomised controlled trial of respiratory rehabilitation. Lancet 1994;344:1394-7.

2 Wijkstra PJ, TenVergert EM, van Altena R, et al. Long term benefits of rehabilitation at home on quality of life and exercise tolerance in patients with chronic obstructive pulmonary disease. Thorax 1995;50:824-8.

3 Ries AL, Kaplan RM, Limberg TM, et al. Effects of pulmonary rehabilitation on physiologic and psychosocial outcomes in patients with chronic obstructive pulmonary disease. Ann Intern Med 1995;122:823-32.

4 Lacasse Y, Wong E, Guyatt GH, et al. Meta-analysis of respiratory rehabilitation in chronic obstructive pulmonary disease. Lancet 1996;348:1115-9.

5 Griffiths TL, Burr ML, Campbell IA, et al. Results at one year of outpatient multidisciplinary pulmonary rehabilitation: a randomised controlled trial. Lancet 2000; 355:362-8.

6 Singh SJ, Smith DL, Hyland ME, et al. A short outpatient pulmonary rehabilitation programme: immediate and longer term effects on exercise performance and quality of life. Respir Med 1998;92:1146-54.

7 American College of Sports Medicine Guidelines for exercise testing and prescription. 4th ed. Philadelphia: Lea and Febiger, 1991.

8 Guyatt GH, Townsend M, Berman LB, et al. Quality of life in patients with chronic airflow limitation. $\mathrm{Br} \mathcal{F}$ Dis Chest 1987;81:45-54.

9 Redelmeier DA, Guyatt GH, Goldstein RS. Assessing the minimal important difference in symptoms: a comparison of two techniques. F Clin Epidemiol 1996;49:1215-9.

10 Hyland ME, Singh SJ, Sodergren SC, et al. Development of a shortened version of the Breathing Problems Questionnaire suitable for use in a pulmonary rehabilitation clinic: a purpose-specific, disease-specific questionnaire. Quality of Life Res 1998;7:227-33.

11 Singh SJ, Morgan MDL, Scott S, et al. Development of a shuttle walking test of disability in patients with chronic airways obstruction. Thorax 1992;47:1019-24.

12 Singh SJ, Morgan MD, Hardman AE, et al. Comparison of oxygen uptake during a conventional treadmill test and the shuttle walking test in chronic airflow limitation. Eur Respir f 1994;7:2016-20. 